\title{
A Study of Holders Inequality in New Spaces
}

\section{C.R. Bhatta}

Central Department of Mathematics

Tribhuvan University, Kirtipur

Corresponding email: crbhatta@yahoo.com

Abstract: The central attraction of this paper is to prove holders inequality in some new spaces such as

Grand Lebesgue Space $L^{\mathrm{P}}$. Small Lebesgue space $\mathrm{L}^{\mathrm{P})^{\prime}}$ and Banach Lattice space $\mathrm{X}^{\mathrm{P}}$.

\section{Introduction}

The Grand Lebesgue Space $L^{p)}$, small Lebesgue Space $L^{p)^{\prime}}$ and Banach lattice space $X^{p}$ have many application in analysis. The grand Lebesgue spaces were introduced by Iwaniec and Sbordone and the small Lebesgue space were introduced by A. fiorenza. The sole purpose of this paper is to study the Holders inequality in these spaces. The concept of these spaces can be applied in Lorentz space as well.

Definition 1.1 (Grand Lebesgue space, $L^{\mathrm{P})}$ ): Let $\Omega \subset \mathfrak{R}^{\mathrm{n}}, \mathrm{n} \in \mathrm{Z}^{+}$, be a set of Lebesgue measure. Let $|\Omega|<\infty$. The Grand Lebesgue space, denoted by $L^{\mathrm{P})}(\Omega)$ such that

$$
\|\mathrm{f}\| \mathrm{p}^{)}={ }^{1 /(\mathrm{P}-\epsilon)}<\infty
$$

\section{Holders inequality on Grand Lebesgue space, $L^{P)}$}

Let $f \in \mathrm{L}^{\mathrm{P}^{\prime}}, \mathrm{g} \in \mathrm{L}^{\left(\mathrm{P}^{\prime}\right.}$ then

$$
\int \operatorname{fgd} \mathrm{x} \leq\left\|\mathrm{f} \mid \mathrm{p}^{)}\right\| \mathrm{g} \|_{\left(\mathrm{p}^{\prime}\right.}
$$

\section{Proof:}

Let $|\mathrm{g}|=\mathrm{g}_{\mathrm{k}}$ be any decomposition with $\mathrm{g}_{\mathrm{k}} \geq 0, \mathrm{k} \in \mathrm{N}$ and let $\mathrm{f} \in \mathrm{L}^{\mathrm{P})}$. For each $\mathrm{k} \in \mathrm{N}$ and for each 0 $<\in<\mathrm{p}-1$, we have

$$
\begin{aligned}
\int_{\Omega} f g_{k} d x & \leq \int_{\Omega}|f| g_{k} d x \\
& \leq \\
& = \\
& \leq \epsilon^{-1 /(p-\epsilon)}
\end{aligned}
$$

Therefore,

$$
\epsilon^{-1 /(p-\epsilon)}
$$


Which implies

$$
\begin{aligned}
& \leq \\
& \leq \\
& \leq \in^{-1(p-\epsilon)^{\prime}}
\end{aligned}
$$

Hence,

$$
\left.\leq\|\mathrm{f}\|_{\mathrm{p}}\right)\|\mathrm{g}\|_{\left(\mathrm{p}^{\prime}\right.}
$$

Definition 1.3 (Small Lebesgue Space $L^{\mathrm{P})}$ : The Small Lebesgue Space, denoted by $L^{\mathrm{p}}$ ', is the space of functions $\mathrm{g}$ in $\boldsymbol{M}_{\mathrm{o}}$ such that

$$
\|\mathrm{g}\| \mathrm{p}^{)^{\prime}}=\|\psi\| \|^{\left(\mathrm{p}^{\prime}\right.}<\infty
$$

where $\boldsymbol{M}_{\circ}$ is the set of all measurable functions whose values lie in $[-\infty, \infty]$, finite a.e. in $\Omega, \Omega \subseteq \Re^{\mathrm{n}},|\Omega|<\infty$.

\subsection{Holders inequality in small Lebesgue space $L^{p)^{\prime}}$}

Let $1<\mathrm{p}<\infty$ and $\Omega \subseteq \mathfrak{R}^{\mathrm{n}}, \mathrm{n} \in \mathrm{Z}^{+},|\Omega|<\infty$. then, for $\mathrm{f} \in \mathrm{L}^{\mathrm{p})^{\prime}}$ and $\mathrm{g} \in \mathrm{L}^{\mathrm{P})^{\prime}}$,

$$
\int_{\Omega} \mathrm{fg} \mathrm{dx} \leq\|\mathrm{f}\|_{\mathrm{p}}{ }^{\mathfrak{s}}\|\mathrm{g}\|_{\mathrm{p})^{\prime}}
$$

Proof:

For any $\mathrm{f} \in \mathrm{L}^{\mathrm{p})}$ and $\mathrm{g} \in \boldsymbol{\mathcal { M }}_{\mathrm{o}}$ in view of 1.3

$$
\begin{aligned}
\int_{\Omega} \mathrm{fg} d \mathrm{~d} & \leq \int_{\Omega}|\mathrm{f}||\mathrm{g}| \mathrm{dx} \\
& =\int_{\Omega}|\mathrm{f}| \psi \mathrm{dx} \\
& =\int_{\Omega}|\mathrm{f}| \psi \mathrm{d} \|_{\left(\mathrm{p}^{\prime}\right.} \\
& =\|\mathrm{f}\|_{\mathrm{p})}\|\psi\|_{\left(\mathrm{p}^{\prime}\right.} \\
& =\|\mathrm{f}\|_{\mathrm{p})}\|\mathrm{g}\|_{\mathrm{p})^{\prime}}
\end{aligned}
$$

\subsection{Definition (Banach Lattice)}

Let $(\Omega, \Sigma, \mu)$ be $\sigma$ finite measures space. Let $\mathrm{L}^{\circ}(\Omega)$ be the space of all extended scalar valued $\mu$ measurable function defined on $\Omega$ and $\mu-$ a.e. on finite on $\Omega$. then, a Banach space X $=\left(\mathrm{X},\|\cdot\|_{\mathrm{x}}\right)$ of $\mathrm{L}^{\circ}(\Omega)$ is called a Banach lattice on $(\Omega, \Sigma, \mu)$ if for every $\mathrm{x} \in \mathrm{X}$, the following implication holds:

$$
\mathrm{y} \in \mathrm{L}^{\mathrm{o}}(\Omega),|\mathrm{y}| \leq|\mathbf{x}| \mu-\text { a.e. } \Rightarrow \mathrm{y} \in \mathrm{X} \text { and }\|\mathrm{y}\|_{\mathrm{x}}=\|\mathbf{x}\|_{\mathrm{x}} .
$$

\subsection{Definition (Banach Lattice)}

Let $\mathrm{X}$ be a Banach Lattice on $(\Omega, \Sigma, \mu)$ and let $-\infty<\mathrm{p}<\infty, \mathrm{p} \neq 0$. The space $\mathrm{X}^{\mathrm{p}}$ consists of all $\mathrm{x} \in$ $\mathrm{L}^{\mathrm{p}}(\Omega)$ such that $\left|\mathbf{x}^{\mathrm{p}}\right| \in \mathrm{X}$ and that 


$$
\|\mathbf{x}\| \mathrm{X}^{\mathrm{p}}=(\|\mathbf{x}\|)^{1 / \mathrm{p}}<\infty
$$

For the case $\mathrm{p}<0$, we assume that

$$
\mathbf{x}=\mathbf{x}(\mathrm{t}) \neq 0 \text { for all } \mathrm{t} \in \Omega
$$

\subsection{Generalized Holders inequality in $\mathrm{X}^{\mathrm{p}}$ space}

Let $\mathbf{x}_{\mathrm{i}} \in \mathrm{x}^{\mathrm{pi}}, 0<\mathrm{p}_{\mathrm{i}}<\infty, \mathrm{i}=1,2, \ldots, \mathrm{N}$. Then

$$
\left\|\prod_{i=1}^{N} \boldsymbol{x}_{i}\right\|_{x^{r}} \leq \prod_{i=1}^{N}\left\|\mathbf{x}_{\mathrm{i}}\right\| \mathrm{X}^{\mathrm{pi}}
$$

Proof:

Let us assume that $\left\|\mathbf{x}_{i}\right\| X^{\mathrm{pi}} \neq 0, \mathrm{i}=1,2, \ldots, \mathrm{N}$, otherwise the assertion is trivial.

Let us put, $\mathrm{y}_{\mathrm{i}}=, \mathrm{i}=1,2, \ldots, \mathrm{N}$

And $\Omega_{\mathrm{o}}=\left\{\mathrm{t} \in \Omega: \prod_{i=1}^{N} \mathrm{y}_{\mathrm{i}}(\mathrm{t}) \neq 0\right\}$

Since $=1$ and the function $\mathrm{f}(\mathrm{u})=\exp (\mathrm{u})$ is convex, for every $\mathrm{t} \in \Omega_{\mathrm{o}}$, we have

$$
\begin{aligned}
\prod_{i=1}^{N}\left|g_{i}(\mathrm{f})\right|^{\mathrm{r}} & =\exp \left(\mathrm{r} \log \prod_{i=1}^{N}\left|\mathrm{~g}_{\mathrm{i}}(\mathrm{t})\right|\right) \\
& =\exp \\
& =\exp \\
& \leq
\end{aligned}
$$

Therefore by using lattice property and the triangle in equality, we have

$$
\| \prod_{i=1}^{N}\left\lfloor y_{i}\|\|_{\mathrm{x}^{r}}^{r} \leq\left\|\mathrm{y}_{\mathrm{i}}\right\|==1\right.
$$

Which implies,

$$
\| \prod_{i=1}^{N}\left\lfloor y_{i} \|_{\mathrm{x}^{\prime \prime}} \leq 1\right.
$$

Hence, the assertion follows.

\section{REFERENCES}

1. A. Fiorenza, Duality and reflexivity in Grand Lebesgue spaces, collectanea Math, 51, 2(2000), 131 148

2. Beckenbach, E.F., Bellman, R. (1975). An introduction to inequalities, Random House Inc. ISBN 0 $394-01559-2$.

3. L. Maligranda and L.E. Persson (1987) on the duality of Banach Lattices, Mathematical Report, Lulea University.

4. S. Banic, J. Pecaric, S. Varosanec (2008), Superquadratic functions and refinements of some classical inequalities. J. Korean Math. Soc. 45, $513-525$. 
